\title{
Ko-translationale Assemblierung von Proteinkomplexen
}

KAI FENZL, GÜNTER KRAMER, BERND BUKAU

ZENTRUM FÜR MOLEKULARE BIOLOGIE DER UNIVERSITÄT HEIDELBERG (ZMBH) UND

DEUTSCHES KREBSFORSCHUNGSZENTRUM (DKFZ), DKFZ-ZMBH ALLIANZ, HEIDELBERG

\section{The majority of cellular proteins exerts their biological activity as oli- gomeric complexes. The general view was that complexes form by random collision of folded subunits in the cytosol. Recent studies question this view by demonstrating that a surprisingly high number of complexes are formed during translation. Co-translational assembly occurs by interaction either of fully synthesized subunits with nascent partner subunits, or of two nascent polypeptides exposed by proximal ribosomes.}

DOI: $10.1007 / \mathrm{s} 12268-021-1682-5$

(C) Die Autoren 2021

Schon während der Translation beginnt die Faltung neu synthetisierter Proteine in native dreidimensionale Tertiärstrukturen, wobei einzelne Proteindomänen, oft durch Chaperone assistiert, sukzessive falten. Die Mehrheit der gefalteten Proteine muss jedoch noch zu Quartärstrukturen assemblieren, die aus homomeren oder heteromeren Untereinheiten bestehen. Es wurde generell angenommen, dass sich die beteiligten Untereinheiten über freie Diffusion und zufällige Kollisionen innerhalb der Zelle finden. Während einerseits die Diffusionsgeschwindigkeit von Proteinen in der Zelle hoch genug erscheint, um Proteinkomplexe innerhalb von Sekunden ausbilden zu lassen, exponieren freie Untereinheiten oft hydrophobe Interaktionsflächen, was die Anfälligkeit für Aggregation, proteolytischen Abbau und unspezifische Interaktionen mit anderen Zellproteinen erhöht. Ein Verlust an Effizienz der Proteinassemblierung ist somit höchst problematisch, gerade für wachsende menschliche Zellen, die hunderttausende Proteine pro Minute herstellen. Aktuelle Ergebnisse zeigen, dass die Assemblierung von stabilen Proteinkomplexen in der Regel ko-translational, also während der Synthese der Untereinheiten durch das Ribosom, stattfindet und damit die freie Diffusion aggregationsanfälliger Untereinheiten minimiert. Die ersten
Hinweise auf ko-translationale Assemblierung von Proteinkomplexen stammen bereits aus den 1960er-Jahren, die belegen, dass das Tetramer der bakteriellen $\beta$-Galactosidase seine enzymatische Aktivität erlangt, bevor die Synthese abgeschlossen ist [1]. In den letzten fünf Jahren wurde dieser Prozess mehrfach für Proteine in Bakterien, Bäckerhefe und humane Zellen nachgewiesen [2-7]. Dabei zeigte sich, dass zwei mechanistische Varianten der ko-translationalen Assemblierung von Proteinkomplexen existieren.

\section{Mechanismus 1: Co-Post-Assemblierung}

Bei der Co-Post-Assemblierung genannten Variante assoziiert ein vollständig synthetisiertes, gefaltetes Protein mit einer naszierenden Partneruntereinheit und bildet am translatierenden Ribosom einen dimeren Komplex aus (Abb. 1A). Der Durchbruch zum experimentellen Nachweis dieses Prozesses war die Entwicklung einer Hochdurchsatzmethode, dem selective ribosome profiling (SeRP) [8-10]. SeRP ermöglicht es, Interaktionen eines ausgewählten Proteins, z. B. der Untereinheit eines Proteinkomplexes oder eines Chaperons, mit translatierenden Ribosomen genomweit zu detektieren [8-10]. Dabei werden zunächst alle Ribosomen einer Zelle isoliert, die sich als Polysomen auf translatierten mRNAs aufreihen, gefolgt von einem Nukleaseverdau der mRNA. Dabei schützt jedes Ribosom ein kurzes mRNA Segment vor dem Verdau (ribosome footprint). Anschließend wird die zu untersuchende Untereinheit eines Proteinkomplexes zusammen mit der assoziierten naszierenden Partneruntereinheit und dem translatierenden Ribosom affinititätsgereinigt. Durch das Sequenzieren der ribosome footprints kann ausgelesen werden, mit welcher naszierenden Polypeptidkette die affinitätsgereinigte Proteinuntereinheit ko-translational interagiert. Dadurch kann bestimmt werden, wann die Assemblierung während der Translation beginnt, und somit auch die Länge der naszierenden Polypeptidkette, bei der die Assemblierung anfängt (Abb. 1B). Die ko-translationale Komplexbildung konnte für mehrere heteromere Proteinkomplexe nachgewiesen werden, z. B. für die Fettsäuresynthase der Bäckerhefe [4] und für Transkriptionsfaktoren in menschlichen Zellen [7]. Von zwölf untersuchten Proteinkomplexen der Hefe folgten neun einem ko-translationalen Assemblierungsprozess, und für die drei Fälle, in denen dies nicht beobachtet wurde, war bekannt, dass die Assemblierung durch spezielle Faktoren posttranslational reguliert wird [4]. Die ko-translationale Assemblierung zeigte meist einen gerichteten Verlauf, d. h. ein vollständig synthetisiertes „Protein A“ interagiert mit der naszierenden Kette des „Partnerproteins B“, aber nicht umgekehrt (Abb. 1C). Diese gerichtete Assemblierung korreliert mit dem Grad der Aggregations- und Degradationsanfälligkeit der naszierenden Polypeptidketten. In allen untersuchten Fällen unterdrückt die Bindung der Partneruntereinheiten die Aggregation und Degradation der ko-translational assemblierenden Proteinuntereinheiten. Die Partneruntereinheiten, die als vollständig gefaltete Proteine binden, neigen dagegen nicht zur Aggregation oder Degradation [4]. Daher liegt es nahe anzunehmen, dass dieser Assemblierungsmodus instabile Untereinheiten während der Synthese stabilisiert und damit die Evolution komplex gefalteter Pro- 
A

Co-Post Assemblierung

cis

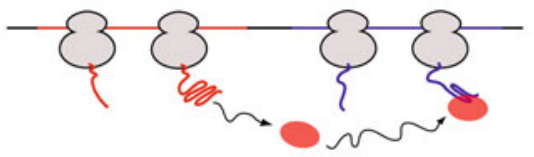

trans
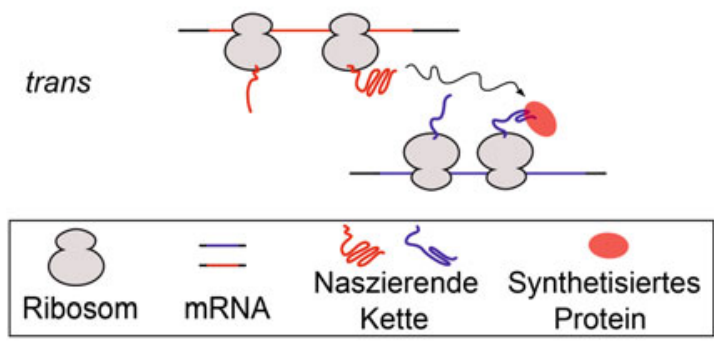

C

IP: $\alpha$-Subunit
B

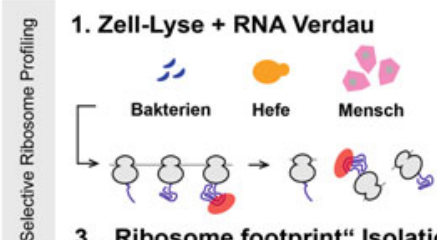

2. Aufreinigung des Zielproteins

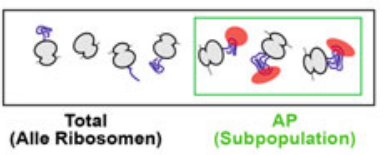

3. „Ribosome footprint" Isolation, Sequenzierung \& Datenanalyse
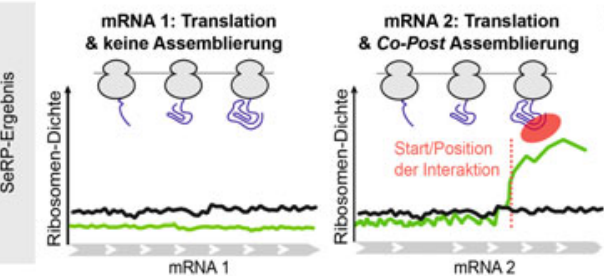

Bekannte Proteinkomplexbildung via Co-Post Assomblierung (in Hofo):

Fettsăuresynthase

Phosphofruktokinase

Aminoacyl-tRNA-Synthetase

Nascent-Polypeptide-Associated Complex (NAC)

IP: $\beta$-Subunit
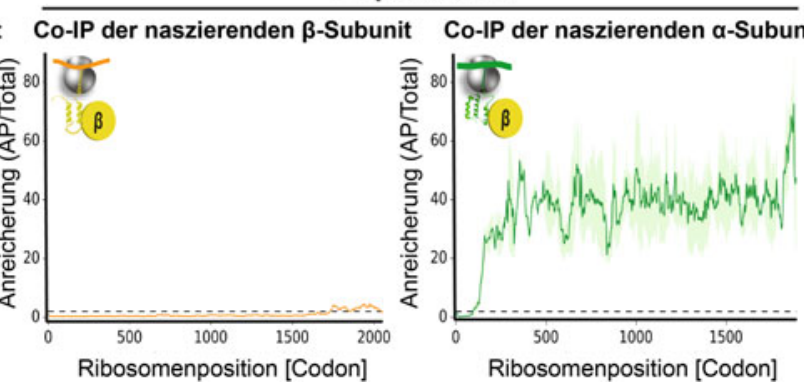

Co-IP der naszierenden $\beta$-Subunit Co-IP der naszierenden $\alpha$-Subunit
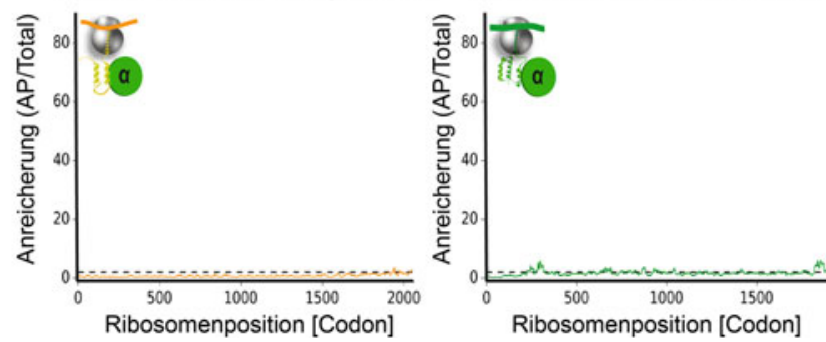

$\Delta$ Abb. 1: Detektion von Co-Post-Assemblierung. A, Co-Post-Assemblierung kann zwischen zwei Ribosomen auf derselben mRNA (cis-Assemblierung, häufig in Prokaryoten) oder auf zwei unterschiedlichen mRNAs (trans-Assemblierung, häufig in Eukaryoten) auftreten. B, SeRP-Methode: Ribosomen werden nach Zelllyse und Ribonukleaseverdau (1) direkt oder nach einer Affinitätsaufreinigung eines Zielproteins (z. B. einer Untereinheit) isoliert (2). Die ribosome footprints beider Fraktionen werden sequenziert und der translatierten Sequenz eines Genes zugeordnet (3). Ein Anstieg spezifischer ribosome footprints in der aufgereinigten Fraktion einer codierenden Sequenz weist auf eine Co-Post-Assemblierung hin. C, SeRP-Analyse des Fettsäuresynthasekomplexes aus Hefe [4]: Die Assemblierung beruht auf der gerichteten Interaktion einer vollständig synthetisierten $\beta$-Untereinheit mit einer naszierenden $\alpha$-Untereinheit. Abbildung in Anlehnung an [4].

teine ermöglicht. Im Einklang mit dieser protektiven Funktion ist der Befund, dass in allen untersuchten Fällen die Bindung der Partneruntereinheit sofort nach dem Austreten der Interaktionsdomäne aus dem ExitTunnel des Ribosoms während der Translation stattfindet. Ko-translational agierende Enzyme und Chaperone müssen daher räumlich und funktional darauf abgestimmt und vermutlich zum Zeitpunkt der Partnerbindung bereits von der naszierenden Peptidkette dissoziiert sein. Dies konnte für das ribosomenassoziierte Hsp70 Chaperon der Hefe, Ssb, nachgewiesen werden [4,11].

Für die ko-translationale Assemblierung von Heterodimeren in Bakterien konnte gezeigt werden, dass die benachbarte Synthese der Interaktionspartner von einer polycistronischen mRNA die Effizienz der Assemblierung erhöht. Die Anordnung der Gene im Operon zeigt, dass das Promoter-proximale (erste) Gen für die Untereinheit codiert, die vollständig synthetisiert und gefaltet die zweite naszierende Partneruntereinheit bindet, die vom Promoter-distalen (zweiten) Gen codiert wird [3]. Es besteht also eine Direktionalität nicht nur bei der Assemblierung, sondern auch beim genetischen Aufbau der codierenden Operons. Wie Co-Post-Assemblierung in Eukaryoten räumlich organisiert ist, in denen Operons selten und die mRNAs stattdessen getrennte Einheiten sind, ist Gegenstand aktueller Forschung. Erste Studien zu einzelnen Komplexen zeigen, dass es in eukaryotischen Zellen zur Ko-Lokalisation der codierenden mRNAs kommen kann, wodurch die freie Diffusion beteiligter Untereinheiten minimiert wird [6,7].

\section{Mechanismus 2: \\ Co-Co-Assemblierung}

Die Erkenntnis, dass Komplexbildung schon während der Translation direkt am Ribosom stattfinden kann, führt zu der Frage, ob auch zwei naszierende Polypeptidketten miteinander interagieren können (Co-Co-Assem- blierung, Abb. 2A). Zwar existierten erste Hinweise für diese Art der Komplexbildung [5-7,12, 13], aber bis vor kurzem fehlten direkte Beweise für die Existenz und Häufigkeit eines solchen Mechanismus. Um diese Fragen zu beantworten wurde disome selective profiling (DiSP) entwickelt, eine proteomweite Detektionsmethode speziell für Co-Co-Assemblierung [5]. Dabei werden Ribosomenpaare (Disomen) isoliert, die durch die Interaktion der naszierenden Polypeptidketten auch nach einem Nukleaseverdau zusammengehalten werden. Danach werden die ribosome footprints der Disomen sequenziert und jedem Gen sequenzspezifisch zugeordnet (Abb. 2B). Damit kann bestimmt werden, welche mRNAs von Ribosomen translatiert werden, die während der Translation durch $\mathrm{Co}^{-} \mathrm{Co}^{-}$ Assemblierung der naszierenden Protein Disomen bilden. Darüber hinaus kann bestimmt werden wann während der Translation die Dimerbildung erfolgt. Die Daten 
cis
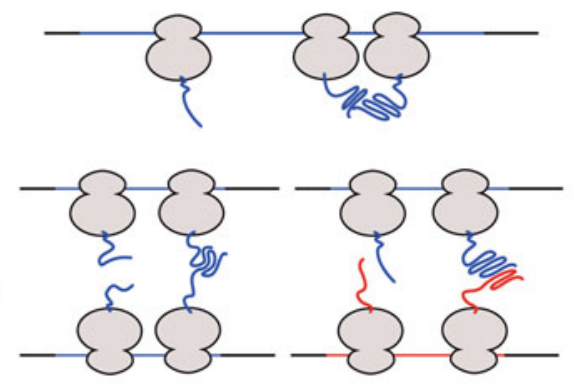

trans

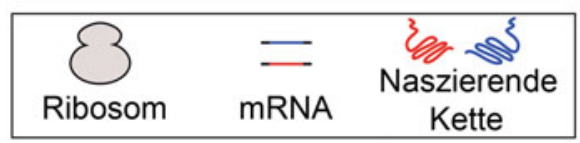

B

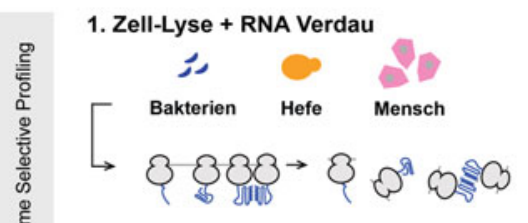

2. Separation der Monsomen und Disomen

3. „Ribosome footprint“" Isolation, Sequenzierung \& Datenanalyse
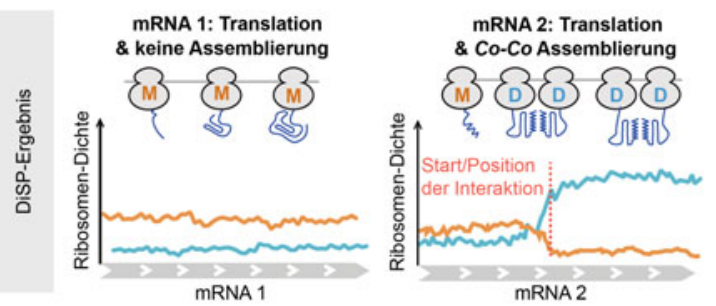

Bekannte Proteinkomplexbildung via Co-Co Assemblierung (in Menschen):

Lamin AVC

Lamin B1 \& B2

Dynactin Subunit 1

$\mathrm{NF}-\mathrm{kB}$

$\Delta$ Abb. 2: Detektion von Co-Co-Assemblierung. A, Co-Co-Assemblierung kann zwischen zwei Ribosomen auf derselben mRNA (cis-Assemblierung) oder auf zwei unterschiedlichen mRNAs (trans-Assemblierung) auftreten und dabei zu Komplexbildung von Homo- wie auch Heteromeren führen. B, DiSP-Methode: Einzelne Ribosomen (Monosomen/M) und gekoppelte Ribosomenpaare (Disomen/D) werden nach der Zelllyse und einem RNA-Verdau (1) in einem Sucrose-Gradienten voneinander separiert (2). Die ribosome footprints werden von beiden Fraktionen aufgereinigt und sequenziert (3). Ein Wechsel translatierender Ribosomen (und damit der ribosome footprints) von der Monosomen-Fraktion (orange) zur Disomen-Fraktion (blau) weist auf Co-Co-Assemblierung hin. Abbildung in Anlehnung an [5].

aus menschlichen Zellen zeigen, dass Hunderte Homodimere durch Interaktion naszierender Polypeptidketten gebildet werden, was über 30 Prozent der bekannten humanen Homooligomere ausmacht [5]. Die häufigste Form der Dimerisierung über $\mathrm{Co}$-Co-Assemblierung erfolgt über die Bildung von coiled coils, bei der sich zwei $\alpha$-Helices zu einem Dimer verbinden. Erste Befunde weisen darauf hin, dass die $\mathrm{Co}-\mathrm{Co}$-Assemblierung von Homodimeren wahrscheinlich zwischen benachbarten Ribosomen auf einer mRNA stattfindet (cis-Assemblierung), was zu einer diffusionsunabhängigen
Assemblierung führt. Ähnlich wie der $\mathrm{Co}$ Post-Assemblierungsmodus ist auch $\mathrm{Co}^{-}$ Co-Assemblierung in der Lage, Aggregation und Degradation nicht assemblierter Untereinheiten zu reduzieren und damit die Effizienz der Proteinkomplexbildung zu erhöhen. Darüber ermöglicht der $\mathrm{Co}^{-}$ Co-Mechanismus in cis auch die spezifische Assemblierung von isoformspezifischen Homodimeren, da gehäuft Proteine in mehreren Isoformen in der Zelle existieren, die durch alternatives Splicing oder Genduplikation entstehen, sich aber trotz identischer Interaktionsdomänen in Zellen nicht mischen. Co-Co-Assemblie- 
rung verhindert damit die Bildung unerwünschter Heterodimere.

\section{Ausblick}

Neue Forschungsergebnisse belegen, dass die ko-translationale Assemblierung von Proteinkomplexen von Bakterien bis zu menschlichen Zellen ein weit verbreiteter Mechanismus ist. Die Ergebnisse erklären, wie Zellen die Effizienz der Komplexbildung im dicht gepackten Cytosol aufrechterhalten und Aggregation, Abbau sowie ungewollte Interaktionen zwischen Proteinen verhindern können. Faltung und Assemblierung finden daher koordiniert an der Oberfläche des translatierenden Ribosoms statt. Diese Ergebnisse eröffnen viele neue und faszinierende Fragestellungen, wie z. B.: Findet die Assemblierung größerer Proteinkomplexe, die aus mehr als zwei Untereinheiten bestehen, auch ko-translational statt? Wie wird eine ausreichende räumliche Nähe zwischen Ribosomen auf einer mRNA bewerkstelligt, und inwieweit wird für die Koordinierung der beteiligten Ribosomen die Translationsgeschwindigkeit lokal verändert? Existiert eine zelluläre Maschinerie, die ko-translationale Prozesse organisiert und reguliert? Bilden sich Heterodimere auch über $\mathrm{Co}-\mathrm{Co}$ Assemblierung und wie wird die dafür notwendige Kolokalisation der beteiligten mRNAs im Cytosol eukaryotischer Zellen gewährleistet? Die Verfügbarkeit der notwendigen Technologien erlauben es jetzt, diese grundlegenden Fragen experimentell anzugehen.

\section{Literatur}

[1] Zipser D (1963) Studies on the ribosome-bound $\beta$-galactosidase of Escherichia coli. J Mol Biol 7: 739-751

[2] Duncan CDS, Mata J (2011) Widespread cotranslational formation of protein complexes. PLoS Genet 7: e1002398

[3] Shieh YW, Minguez P, Bork P et al. (2015) Operon structure and cotranslational subunit association direct protein assembly in bacteria. Science 350: 678-680

[4] Shiber A, Döring K, Friedrich U et al. (2018)

Cotranslational assembly of protein complexes in eukaryotes revealed by ribosome profiling. Nature 561: 268-272
[5] Bertolini M, Fenzl K, Kats I et al. (2021) Interactions between nascent proteins translated by adjacent ribosomes drive homomer assembly. Science 371: 57-64

[6] Panasenko OO, Somasekharan SP, Villanyi Z et al. (2019) Co-translational assembly of proteasome subunits in NOT1containing assemblysomes. Nat Struct Mol Biol 26: 110-120 [7] Kamenova I, Mukherjee P, Conic S et al. (2019) Co-translational assembly of mammalian nuclear multisubunit complexes. Nat Commun 10: 25-28

[8] Oh E, Becker AH, Sandikci A et al. (2011) Selective ribosome profiling reveals the cotranslational chaperone action of trigger factor in vivo. 147: 1295-1308

[9] Becker AH, Oh E, Weissman JS et al. (2013) Selective ribosome profiling as a tool for studying the interaction of chaperones and targeting factors with nascent polypeptide chains and ribosomes. Nat Protoc 8: 2212-2239 [10] Galmozzi CV, Merker D, Friedrich UA et al. (2019) Selective ribosome profiling to study interactions of translat ing ribosomes in yeast. Nat Protoc 14: 2279-2317

[11] Döring K, Ahmed N, Riemer T et al. (2017) Profiling Ssb-nascent chain interactions reveals principles of Hsp70assisted folding. Cell 170: 298-311

[12] Nicholls CD, Mclure KG, Shields MA, Lee PWK (2002) Biogenesis of p53 involves cotranslational dimerization of monomers and posttranslational dimerization of dimers. J Biol Chem 277: 12937-12945

[13] Lin L, DeMartino GN, Greene WC (2000) Cotranslational dimerization of the Rel homology domain of NF-kappaB1 generates p50-p105 heterodimers and is required for effective p50 production. EMBO J 19: 4712-4722
Funding note: Open Access funding enabled and organized by Projekt DEAL. Open Access: Dieser Artikel wird unter der Creative Commons Namensnennung 4.0 International Lizenz veröffentlicht, welche die Nutzung, Vervielfältigung,
Bearbeitung, Verbreitung und Wiedergabe in jeglichem Medium und Format Bearbeitung, Verbreitung und Wiedergabe in jeglichem Medium und
erlaubt, sofern Sie den/die ursprünglichen Autor(en) und die Quelle ordnungsgemäß nennen, einen Link zur Creative Commons Lizenz beifügen und angeben, ob Änderungen vorgenommen wurden. Die in diesem Artikel enthaltenen Bilder und sonstiges Drittmaterial unterliegen ebenfalls der genannten Creative Commons Lizenz, sofern sich aus der Abbildungslegende nichts anderes ergibt. Sofern das betreffende Material nicht unter der genannten Creative Commons Lizenz steht und die betreffende Handlung nich nach gesetzlichen Vorschriften erlaubt ist, ist fur die oben aufgefuhin Weiterverwendungen des Materials die Einwilligung des jeweiligen Recheinhabers einzuholen. Weilere Details zur Lizenz entnehmen Sie bitte der
Korrespondenzadresse:

Prof. Dr. Bernd Bukau

Zentrum für Molekulare Biologie der Universität Heidelberg (ZMBH)

Im Neuenheimer Feld 282

D-69120 Heidelberg

bukau@zmbh.uni-heidelberg.de

\begin{tabular}{|c|c|}
\hline \multicolumn{2}{|l|}{ AUTOREN } \\
\hline & $\begin{array}{l}\text { Kai Fenzl } \\
\text { Jahrgang } 1991 \text {. Bachelor of Science } 2014 \text { an der TU-Darmstadt und Master of } \\
\text { Science } 2016 \text { an der Universität Heidelberg. 2017-2021 Promotion im Labor von } \\
\text { Prof. Dr. B. Bukau am Zentrum für Molekular Biologie der Universität Heidelberg } \\
\text { (ZMBH). Aktuell Postdoc am European Molecular Biology Laboratory (EMBL) im Labor } \\
\text { von Prof. Dr. L. Steinmetz. }\end{array}$ \\
\hline & $\begin{array}{l}\text { Günter Kramer } \\
\text { Jahrgang 1968. Biologiestudium in Konstanz. } 1999 \text { Promotion. 2000-2003 Postdoc } \\
\text { am Institut für Biochemie der Universität Freiburg und dem Zentrum für Molekulare } \\
\text { Biologie Heidelberg (ZMBH), Universität Heidelberg. 2003-2005 Postdoc am White- } \\
\text { head Institute, Massachusetts Institute of Technology in Cambridge, USA. Seit } 2005 \\
\text { Projektleiter am ZMBH, Universität Heidelberg. }\end{array}$ \\
\hline & $\begin{array}{l}\text { Bernd Bukau } \\
\text { Biologiestudium und Promotion. 1989-1997 Leiter einer Nachwuchsgruppe am } \\
\text { ZMBH. 1997-2002 C4-Professor für Biochemie am Institut für Biochemie der Medizi- } \\
\text { nischen Fakultät der Universität Freiburg und von 1999-2001 Direktor des Instituts. } \\
\text { Seit } 2002 \text { C4/W3-Professor für Molekulare Biologie am ZMBH der Universität Heidel- } \\
\text { berg und Direktor des ZMBH von 2005-2018. Seit 2011 Leiter einer Brückenabtei- } \\
\text { lung am Deutschen Krebsforschungszentrum (DKFZ) Heidelberg und seit } 2008 \\
\text { Ko-Direktor der DKFZ-ZMBH-Allianz. }\end{array}$ \\
\hline
\end{tabular}

